\title{
The impact of slice-reduced computed tomography on histogram- based densitometry assessment of lung fibrosis in patients with systemic sclerosis
}

\author{
Thi Dan Linh Nguyen-Kim ${ }^{1}$, Britta Maurer ${ }^{2}$, Yossra A. Suliman ${ }^{2,3}$, Fabian Morsbach ${ }^{1}$, Oliver Distler ${ }^{2 *}$, \\ Thomas Frauenfelder ${ }^{1 *}$
}

${ }^{1}$ Institute of Diagnostic and Interventional Radiology, ${ }^{2}$ Division of Rheumatology University Hospital Zurich, Raemistrasse, Zurich, Switzerland; ${ }^{3}$ Department of Rheumatology and Rehabilitation, Faculty of Medicine, Assuit University Hospital, Assuit, Arab Republic of Egypt

Contributions: (I) Conception and design: TDL Nguyen-Kim, T Frauenfelder, O Distler; (II) Administrative support: T Frauenfelder, O Distler; (III) Provision of study materials or patients: TDL Nguyen-Kim, T Frauenfelder, O Distler, B Maurer, YA Suliman; (V) Data analysis and Interpretation: TDL Nguyen-Kim, F Morsbach, T Frauenfelder, O Distler, B Maurer, YA Suliman; (VI) Manuscript writing: All authors; (VII) Final approval of manuscript: All authors.

*These authors contributed equally to this work.

Correspondence to: Thi Dan Linh Nguyen-Kim, MD. Institute of Diagnostic and Interventional Radiology, University Hospital Zurich, Raemistrasse 100, CH-8091 Zurich, Switzerland. Email: thidanlinh.nguyen@usz.ch.

Background: To evaluate usability of slice-reduced sequential computed tomography (CT) compared to standard high-resolution CT (HRCT) in patients with systemic sclerosis (SSc) for qualitative and quantitative assessment of interstitial lung disease (ILD) with respect to (I) detection of lung parenchymal abnormalities, (II) qualitative and semiquantitative visual assessment, (III) quantification of ILD by histograms and (IV) accuracy for the $20 \%$-cut off discrimination.

Methods: From standard chest HRCT of 60 SSc patients sequential 9-slice-computed tomography (reduced HRCT) was retrospectively reconstructed. ILD was assessed by visual scoring and quantitative histogram parameters. Results from standard and reduced HRCT were compared using non-parametric tests and analysed by univariate linear regression analyses.

Results: With respect to the detection of parenchymal abnormalities, only the detection of intrapulmonary bronchiectasis was significantly lower in reduced HRCT compared to standard HRCT (P=0.039). No differences were found comparing visual scores for fibrosis severity and extension from standard and reduced HRCT $(\mathrm{P}=0.051-0.073)$. All scores correlated significantly $(\mathrm{P}<0.001)$ to histogram parameters derived from both, standard and reduced HRCT. Significant higher values of kurtosis and skewness for reduced HRCT were found (both $\mathrm{P}<0.001$ ). In contrast to standard HRCT histogram parameters from reduced HRCT showed significant discrimination at cut-off $20 \%$ fibrosis (sensitivity $88 \%$ kurtosis and skewness; specificity $81 \%$ kurtosis and $86 \%$ skewness; cut-off kurtosis $\leq 26$, cut-off skewness $\leq 4$; both $\mathrm{P}<0.001$ ).

Conclusions: Reduced HRCT is a robust method to assess lung fibrosis in SSc with minimal radiation dose with no difference in scoring assessment of lung fibrosis severity and extension in comparison to standard HRCT. In contrast to standard HRCT histogram parameters derived from the approach of reduced HRCT could discriminate at a threshold of $20 \%$ lung fibrosis with high sensitivity and specificity. Hence it might be used to detect early disease progression of lung fibrosis in context of monitoring and treatment of SSc patients.

Keywords: Interstitial lung disease (ILD); reduced high-resolution computed tomography of the lung (reduced HRCT of the lung); histogram parameters from reduced high-resolution computed tomography (histogram parameters from reduced HRCT); systemic sclerosis (SSc)

Submitted Sep 18, 2017. Accepted for publication Mar 22, 2018.

doi: $10.21037 /$ jtd.2018.04.39

View this article at: http://dx.doi.org/10.21037/jtd.2018.04.39 


\section{Introduction}

Pulmonary involvement occurs often in systemic sclerosis (SSc) and is now the leading cause of mortality in SSc patients (1). An autopsy-study showed parenchymal lung abnormalities in up to $80 \%$ of SSc patients (2). Pulmonary function tests are considered the gold standard for the screening of parenchymal lung involvement. However, because lung function tests as well as chest $\mathrm{X}$-ray tend to be insensitive to detect early lung involvement, high-resolution computed tomography (HRCT) plays an important role in the detection and characterization of interstitial lung disease (ILD) in patients with SSc (3-8).

According to Lynch and Best, the visual extent of fibrosis on computed tomography (CT) images is the strongest independent predictor of mortality in patients with idiopathic pulmonary fibrosis (IPF) $(5,9)$. Additionally, Hoffmann-Vold et al. showed that HRCT performed at baseline in SSc patients can predict the development of fibrosis (10). Different studies have shown that the extent and severity of ILD can be adequately assessed using visual scales $(3,6,8)$. Further the extension of fibrosis is evidently associated with an increased risk of death (11-13).

During the last decade, several studies evaluated the value of quantitative assessment of ILDs. Especially the skewness (describes the extent of asymmetry of histograms) and the kurtosis (describes how sharply peaked a histogram is) of the Hounsfield unit (HU) histogram derived from CT showed a strong correlation with the visual assessment of lung involvement (14). Quantitative assessment of fibrosis is a proven robust method with the advantage of standardization and of being more objective and readerindependent (14-17). HRCT has the capability (I) to early detect ILD, (II) to quantify the extent of ILD, and (III) to monitor treatment and disease progression (8,18-22).

Because HRCT adds important information in the diagnostic evaluation of patients with suspected ILD, guidelines for the evaluation with HRCT have been developed (5). However, the use of CT for screening and follow-up has to be considered carefully due to the accumulating radiation dose with an increase of the risk of cancer (23-25). For daily routine, a method has to be found and validated that balances between the high diagnostic sensitivity of CT and the radiation dose $(1,24,26)$. Sverzellati et al. could show that low-dose spiral CT with reduced milliampere $(\mathrm{mA})$ allows successful quantitative evaluation of lung fibrosis in patients with idiopathic interstitial pneumonitis (16). Pontana et al. could show, that with a dose reduction of $60 \%$, CT images reconstructed with sinogramm-affirmed iterative reconstruction (SAFIRE) still allow a similar detection of ILD in SSc patients (27). Besides different scan parameter based dose modulations, reduction of scan range or number of images per scan can also lead to a significant dose reduction maintaining the high diagnostic sensitivity $(16,28)$.

An alternative strategy is the reduction of scan range or the number of slices by applying a sequential mode. A former prospective study by Winklehner et al. showed that the use of a dedicated HRCT based on reduced sequential slices throughout the entire chest can detect lung fibrosis with a high diagnostic sensitivity and accuracy of both $94 \%$ with a very low radiation dose (28). Similar strategies with the aim of dose reduction in CT for other indications have also been performed by Bankier et al. and de Jong et al. $(29,30)$. The advantage of a low sampling protocol is the very low radiation dose of less than $0.028 \mathrm{mSv}$, which is in the range of a chest $\mathrm{X}$-ray (28). Further Frauenfelder $e t a l$. could confirm in a retrospective image analysis that by the approach of using a sequential reduced 9-slice HRCT mild ILD can reliably be detected with the advantage of a much lower radiation dose (31).

To date the differences between a sequential reduced sliced HRCT and standard HRCT in context of assessment of lung fibrosis and the quantitative histogram-based approach has not been evaluated in detail.

The aim of this study was to retrospectively evaluate the usability of slice-reduced sequential CT [further named reconstructed sequential 9-slice-computed tomography (reduced HRCT)] compared to standard HRCT in patients with SSc with respect to (I) detection of lung parenchymal abnormalities, (II) qualitative and semiquantitative visual assessment, (III) quantification of ILD by histograms and (IV) accuracy for the threshold of $20 \%$ lung fibrosis discrimination.

\section{Methods}

\section{Study population}

Sixty patients [median age 61.5 years (range, 28-77 years), 44 females, 16 males] were included for this study with the following inclusion-criteria: All patients fulfilled the very early diagnosis of SSc (VEDOSS) and/or the American College of Rheumatology (ACR) classification criteria for SSc (32-34). All patients underwent HRCT during a standardized assessment program, 
which also included clinical examination, lung function tests, echocardiography and laboratory analysis based on European Scleroderma Trials and Research group (EUSTAR) recommendations (32). Of the enrolled patients, 33 had limited SSc and 27 patients had diffuse SSc based on the LeRoy criteria (4). Patient recruitment and assessment of clinical parameters was performed prospectively, the image analysis retrospectively. The study was approved as part of the SSc registry by the local ethical committee. All patients signed informed consent.

\section{CT protocol for standard HRCT and reconstruction of reduced HRCT}

All CT-images were acquired in prone position in full inspiration. CT scans were performed with two types of scanners: 128 slice multidetector CT scanner (Somatom Flash, Siemens, Erlangen, Germany) and 64 slice multidetector CT scanner (Somatom AS, Siemens, Erlangen, Germany). The CT protocol included a topogram and one serie in prone position. The following parameters were used for the standard-HRCT: Tube voltage $120 \mathrm{kV}$, tube current $30 \mathrm{mAs}$ (reference dose, care dose: on), slice thickness: $1 \mathrm{~mm}$, increment: $0.8 \mathrm{~mm}$, kernel $\mathrm{B} 30$ and B70. The standard HRCT was reconstructed with iterative reconstruction (SAFIRE) strength 3 and 5. Using the standard HRCT dataset a 9-slice-CT (further named reduced HRCT) was reconstructed as following: nine axial slices with an apico-basal gradient, whereas the first three slices had an increment of about $80 \mathrm{~mm}$ (first slice: manubrium sterni, second slice: hilum, third slice: level of lower lobe, based on increment between first and second slice); for the following 6 slices the increment was $15 \mathrm{~mm}$ (covering the lung basis) (28). To simulate the reproducibility of reduced HRCT retrospectively in a possible prospective setting, in a subgroup of 20 patients slightly different 9 slices with a systematic distance difference within a range of $1-3 \mathrm{~cm}$ in comparison to the aforementioned standardized slice levels but with the same defined increment between the slices within the same specimen was reconstructed.

\section{Radiation dose estimation}

One patient was scanned with the aforementioned standardized reduced 9 slices (reduced HRCT) series to estimate the radiation dose reduction compared to standard HRCT (35).

\section{Visual analysis}

All standard HRCT and reduced HRCT were independently assessed by two radiologists (TF, 12 years of experience; BD, 5 years of experience) on a PACS workstation (Impax, AGFA, Dübendorf, Switzerland) with a fixed window/level setting (window: 1,600 HU, level: $-600 \mathrm{HU}$ ).

The visual analysis included the assessment of severity (qualitative assessment) and extension (semiquantitative assessment), based on previous publications: the severity score is based on definition of five parenchymal abnormalities assumed to reflect the increasing severity of lung involvement. It ranges from 0 to 15 and has been adapted from Camiciottoli et al. (14): ground-glass appearance (score 1), irregular pleural margins (score 2), septal and subpleural lines (score 3), honeycombing (score 4), and subpleural cysts (score 5) (Table S1).

The extension score was adapted from Goldin et al. (3): each lung was divided into three zones: upper zone (from apex to upper level of carina), middle zone (from upper part of carina to division of main lower lobe bronchus into segmental bronchi) and lower zone (from the division of main lower lobe bronchus into segmental bronchi to the basis of the lung). Each zone was evaluated using a scale from 0 to 4 (0 to 4 ; 0 , no fibrosis, $4,>75 \%$ of fibrosis) (Table S2). Additionally, the sum of both scores (the severity and extension score) was calculated, further named total score. In order to prevent a recall-bias an interval of 1 month was set between both readouts.

\section{Quantitative analysis}

The lung density histogram parameters including mean lung attenuation (mean HU derived from lung segmentation), kurtosis and skewness were calculated from the standard HRCT and the reduced HRCT, using dedicated commercially available software (myrian, intrasense, France). The kurtosis is a value for sharpness of histogram peak. A lower value means a more platykurtic (flat and lower) curve with a more homogenous distribution of the histogram parameters. The skewness is a value for asymmetry of distribution in HU-histogram. A higher value means a more asymmetric distribution, whereas a positive value represents a positively skewed or left-skewed distribution (distribution towards higher HU-values). 
Table 1 Clinical characteristics

\begin{tabular}{|c|c|}
\hline Items & Clinical characteristics \\
\hline Age (years), mean $\pm S D$ & $58 \pm 13$ \\
\hline $\operatorname{Sex}(n)$ & Male $(n=16)$, female $(n=44)$ \\
\hline $\begin{array}{l}\text { Disease duration (months), } \\
\text { median (range) }\end{array}$ & $81(10-564)$ \\
\hline Disease subsets (n) & ISSc $(n=33)$, dSSc $(n=27)$ \\
\hline $\begin{array}{l}\text { Extent of skin fibrosis } \\
\text { assessed by the modified } \\
\text { Rodnan Skin score, } \\
\text { median (range) } / 51\end{array}$ & $9.5(0-31) / 51$ \\
\hline Antibody profile (n) & $\begin{array}{l}\text { ANA positive }(n=59) \\
\text { ACA positive }(n=16) \\
\text { Scl-70 positive }(n=23) \\
\text { RNA-polymerase III positive } \\
(n=7)\end{array}$ \\
\hline $\begin{array}{l}\text { Major organ involvement } \\
\text { except lung fibrosis } \pm(n)\end{array}$ & $\begin{array}{l}\text { Heart }(n=18) \\
\text { Gastrointestinal tract }(n=10) \\
\text { Pulmonary arterial hypertension } \\
(n=5)\end{array}$ \\
\hline \multicolumn{2}{|c|}{ Lung function parameters, mean \pm SD (range) } \\
\hline FVC (\%) predicted & $92.49 \pm 22.1(41-136)$ \\
\hline FEV1 (\%) predicted & $89.76 \pm 19.74(42-122.2)$ \\
\hline TLC (\%) predicted & $90.74 \pm 2.83(31.1-139)$ \\
\hline DCLO (\%) predicted & $76.32 \pm 19.3(40-123)$ \\
\hline
\end{tabular}

Visual and quantitative analysis in reduced HRCT with slightly different chosen 9 slices

The visual analysis as well as the quantitative analysis as described in the last two passages were also performed for the reduced HRCT with slightly different chosen 9 slices and compared to the reduced HRCT as well as to the standard HRCT.

\section{Statistics}

For statistical analysis, SPSS Version 22 was used. The McNemar-test was applied to compare the qualitative assessment of the parenchymal lung abnormalities. Univariate linear regression analyses were used to correlate the relationship of histogram parameters with the severity score, the extension score and the total score. Based on the results of Goh et al. $(12,36)$ showing that a threshold of $20 \%$ fibrosis on $\mathrm{CT}$ scan provides a discriminatory prognostic information, two groups were built [group $1(\leq 20 \%$ fibrosis), group 2 (>20\% fibrosis)]. The formation of group 1 and group 2 was based on the fibrosis extension score (Table S2). These groups were correlated with the kurtosis and skewness by regression analyses (12). Interreader agreement for visual assessment was calculated by Krippendorff's alpha (perfect reliability $\alpha=1.00$, good reliability $\alpha>0.800$, acceptable $>0.667$, absence of reliability $\alpha=0.000)(37-39)$. $P$ values $<0.05$ were considered statistically significant.

\section{Results}

\section{Patient characteristics}

The clinical characteristics of the 60 patients recruited for the study are summarized in Table $1(40,41)$. In 31 patients $\leq 20 \%$ lung fibrosis was found, in 29 patients fibrotic lung involvement was $>20 \%$.

\section{Radiation dose estimation}

The estimated effective radiation dose for the reduced HRCT was $0.041 \mathrm{mSv}$ (equivalent to a dose reduction of $97.4 \%$, standard of reference $1.6 \mathrm{mSv}$ ).

\section{Detection of lung pattern abnormalities: standard HRCT versus reduced HRCT}

The overall detection rate of parenchymal abnormalities did not significantly differ between standard and reduced HRCT $(\mathrm{P}=0.063-1.0)$ with exception of bronchiectasis, which were detected at a significantly lower rate on reduced HRCT $(\mathrm{P}=0.039)$ (Table 2). The interreader variability was acceptable to good (Krippendorf's $\alpha=0.68-0.92$ ).

\section{Qualitative and semiquantitative assessment score: standard HRCT versus reduced HRCT}

The qualitative and semiquantitative assessments showed no significant difference between standard HRCT and reduced HRCT $(\mathrm{P}=0.051-0.06)$ but with a trend to a higher severity score and total score in the reduced HRCT (Table 3).

\section{Correlation of fibrosis assessment scores with histogram parameters}

The visually assessed severity score, extension score and total score correlated significantly $(\mathrm{P}<0.001)$ with the 
Table 2 Detection of lung patterns in the standard HRCT versus reduced HRCT

\begin{tabular}{lccc}
\hline \multirow{2}{*}{ Lung pattern } & & Patients with abnormality & P value \\
\cline { 2 - 4 } Ground-glass & Standard HRCT (n, \%) & Reduced HRCT (n, \%) & 1.000 \\
Pleural margins & $15(25 \%)$ & $35(58.3 \%)$ & 0.125 \\
Subpleural lines & $39(65 \%)$ & $40(66.7 \%)$ & 1.000 \\
Honeycombing & $42(70 \%)$ & $7(11.7 \%)$ & 0.063 \\
Emphysema & $10(16.7 \%)$ & $2(3.3 \%)$ & 0.500 \\
Bronchiectasis & $3(5 \%)$ & $12(20 \%)$ & 0.039 \\
\hline
\end{tabular}

HRCT, high-resolution computed tomography.

Table 3 Qualitative and semiquantitative assessment scores derived from standard HRCT and from reduced HRCT

\begin{tabular}{|c|c|c|c|c|c|c|c|}
\hline Assessment score & \multicolumn{3}{|c|}{ Standard HRCT } & \multicolumn{3}{|c|}{ Reduced HRCT } & $P$ value \\
\hline Severity score (qualitative assessment) & 4.68 & 3.49 & $0-15$ & 5.23 & 4.28 & $0-18$ & 0.051 \\
\hline Extension score (semiquantitative assessment) & 4.99 & 5.28 & $0-19$ & 4.83 & 5.02 & $0-21$ & 0.073 \\
\hline $\begin{array}{l}\text { Total score (summary score of severity score and } \\
\text { extension score) }\end{array}$ & 9.61 & 7.93 & $0-25$ & 10.36 & 8.67 & $0-26$ & 0.060 \\
\hline
\end{tabular}

HRCT, high-resolution computed tomography; SD, standard deviation.

skewness and kurtosis for reduced and standard HRCT (reduced HRCT-kurtosis: $\mathrm{r}=0.50-0.54$, skewness: $\mathrm{r}=0.50-0.56$; whole-chest-CT-kurtosis: $\mathrm{r}=0.48-0.54$, skewness: $r=0.49-0.57)$.

Figure 1 demonstrates a representative example of the two different curves derived from the histogram parameters from the standard HRCT and reduced HRCT within the same patient.

\section{Histogram parameters: standard HRCT versus reduced HRCT}

Reduced HRCT revealed significant higher values for mean kurtosis and skewness in comparison to standard HRCT (for both $\mathrm{P}<0.001$, Table 4).

\section{Histogram parameters in correlation with prognostic factors in SSc patients}

For standard HRCT no significant difference was found for the $20 \%$-cutoff ( $\mathrm{P}=0.053$ for kurtosis, $\mathrm{P}=0.061$ for skewness) (Figure $2 A$ ), comparing the histogram parameters between group 1 ( $\leq 20 \%$ fibrosis) and group 2 ( $>20 \%$ fibrosis). For reduced HRCT a differentiation between group $1(\leq 20 \%$ fibrosis) and group 2 ( $>20 \%$ fibrosis) was seen with a sensitivity of $88 \%$ for kurtosis and skewness and a specificity of $81 \%$ for kurtosis and $86 \%$ for skewness (cut-off point kurtosis $\leq 26$, cut-off point skewness $\leq 4)(\mathrm{P}<0.001$ for both, kurtosis and skewness) (Figure 2B). The positive predictive values (PPVs) were 62\% for both and the negative predictive values (NPVs) were $80 \%$ and $78 \%$ for kurtosis and skewness.

\section{Association of lung function parameter with histogram parameters}

Forced vital capacity (FVC) correlated significantly (for both $\mathrm{P}<0.001$ ) with the skewness and kurtosis for reduced and standard HRCT (reduced HRCT-kurtosis: $\mathrm{R}=0.48$, skewness: $\mathrm{R}=0.53$; whole-chest-CT-kurtosis: $\mathrm{R}=0.41$, skewness: $\mathrm{R}=0.40$ ).

\section{Visual and quantitative analysis in reduced HRCT with slightly different chosen 9 slices versus standardized reduced HRCT}

Comparing the reduced HRCT with slightly different 


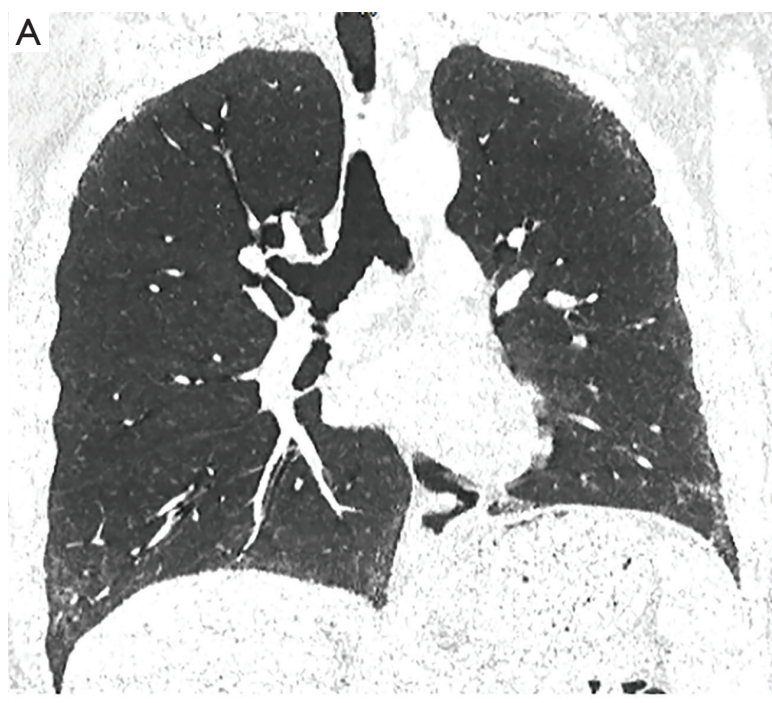

Segmentation of whole lung volume from standard HRCT
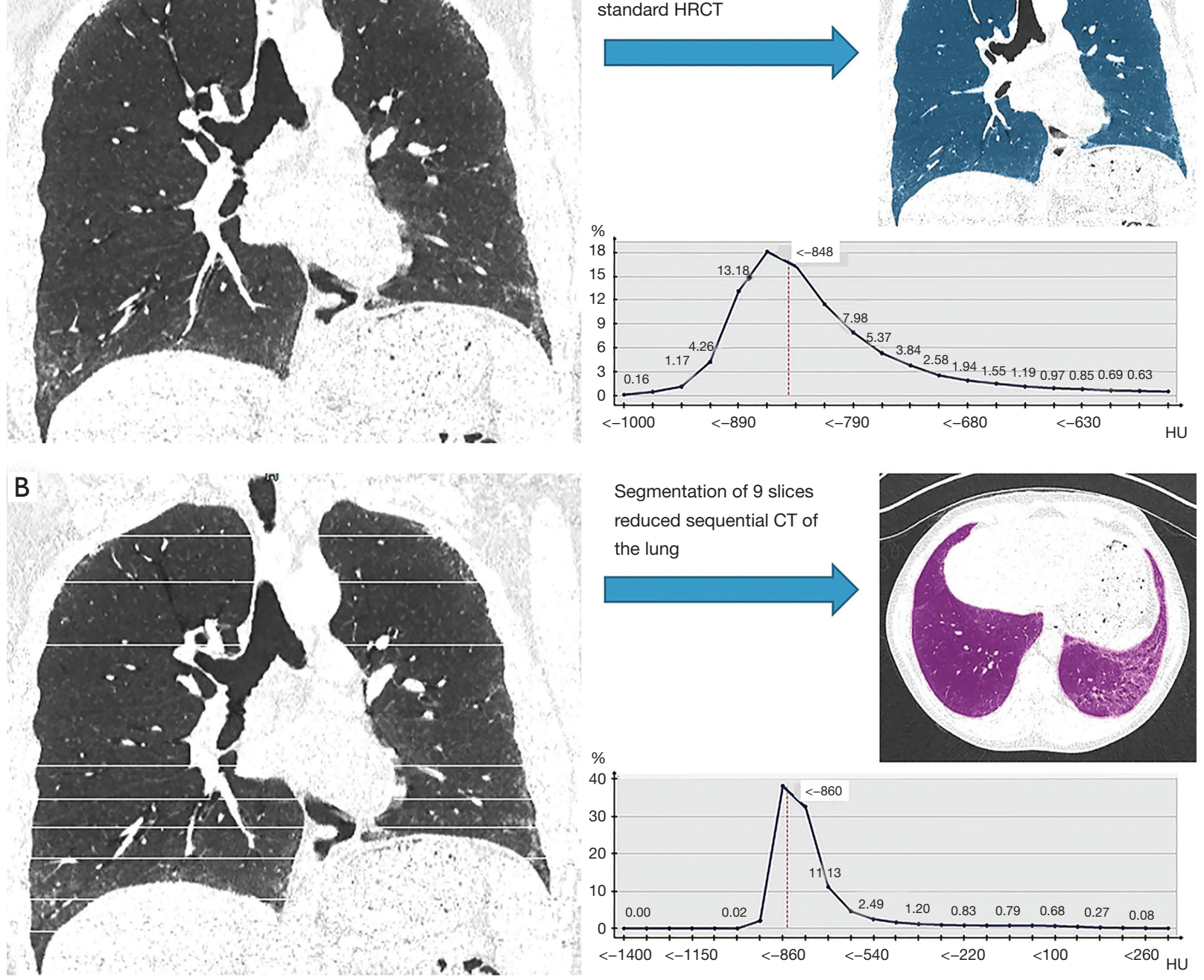

Figure 1 Curve derived from the histogram parameters from the CT of the same patient by (A) standard HRCT chest-CT and (B) reduced HRCT demonstrating the change in the skewness and the kurtosis. CT, computed tomography; HRCT, high-resolution computed tomography.

chosen 9 slices to the standardized reduced HRCT, no significant difference was seen in the detection of lung patterns $(\mathrm{P}>0.871)$, as well as for qualitative assessment of the severity score (mean $4.98 \pm$ SD 4.32 versus mean $5.23 \pm \mathrm{SD} 4.28 ; \mathrm{P}=0.615)$, semiquantitative assessment of the extension score (mean $4.78 \pm$ SD 4.95 versus mean $4.83 \pm \mathrm{SD} 5.02 ; \mathrm{P}=0.086$ ), and the total score (mean $10.05 \pm$ SD 9.07 versus mean $10.36 \pm$ SD 8.67; $\mathrm{P}=0.071$ ).

No significant difference was observed comparing the reduced HRCT with slightly different chosen 9 slices to standardized reduced HRCT for the severity score
( $\mathrm{P}=0.067)$, the extension score $(\mathrm{P}=0.081)$, and the total score $(\mathrm{P}=0.057)$.

The histogram parameters derived from the reduced HRCT with slightly different chosen 9 slices also did not differ significantly in comparison to the standardized reduced HRCT (kurtosis: mean 21.6 \pm SD 9.14 versus mean $22.4 \pm \mathrm{SD}$ 8.66; $\mathrm{P}=0.074$ and skewness: mean $3.7 \pm \mathrm{SD}$ 1.84 versus $3.63 \pm \mathrm{SD} 0.94 ; \mathrm{P}=0.067)$. Also the histogram parameters derived from the reduced HRCT with slightly different chosen 9 slices where significantly higher in comparison to whole standard HRCT (Kurtosis: 21.6 \pm SD 
Table 4 Histogram parameters derived from standard HRCT and from reduced HRCT

\begin{tabular}{|c|c|c|c|c|c|c|c|}
\hline Assessment score & \multicolumn{3}{|c|}{ Standard HRCT } & \multicolumn{3}{|c|}{ Reduced HRCT } & $P$ value \\
\hline Kurtosis & 12.50 & 7.12 & $2.95-40.85$ & 22.4 & 8.66 & $4.73-47.53$ & $<0.001$ \\
\hline Skewness & 2.58 & 0.9 & $0.85-4.80$ & 3.63 & 0.94 & $1.34-5.47$ & $<0.001$ \\
\hline
\end{tabular}

HRCT, high-resolution computed tomography; SD, standard deviation.
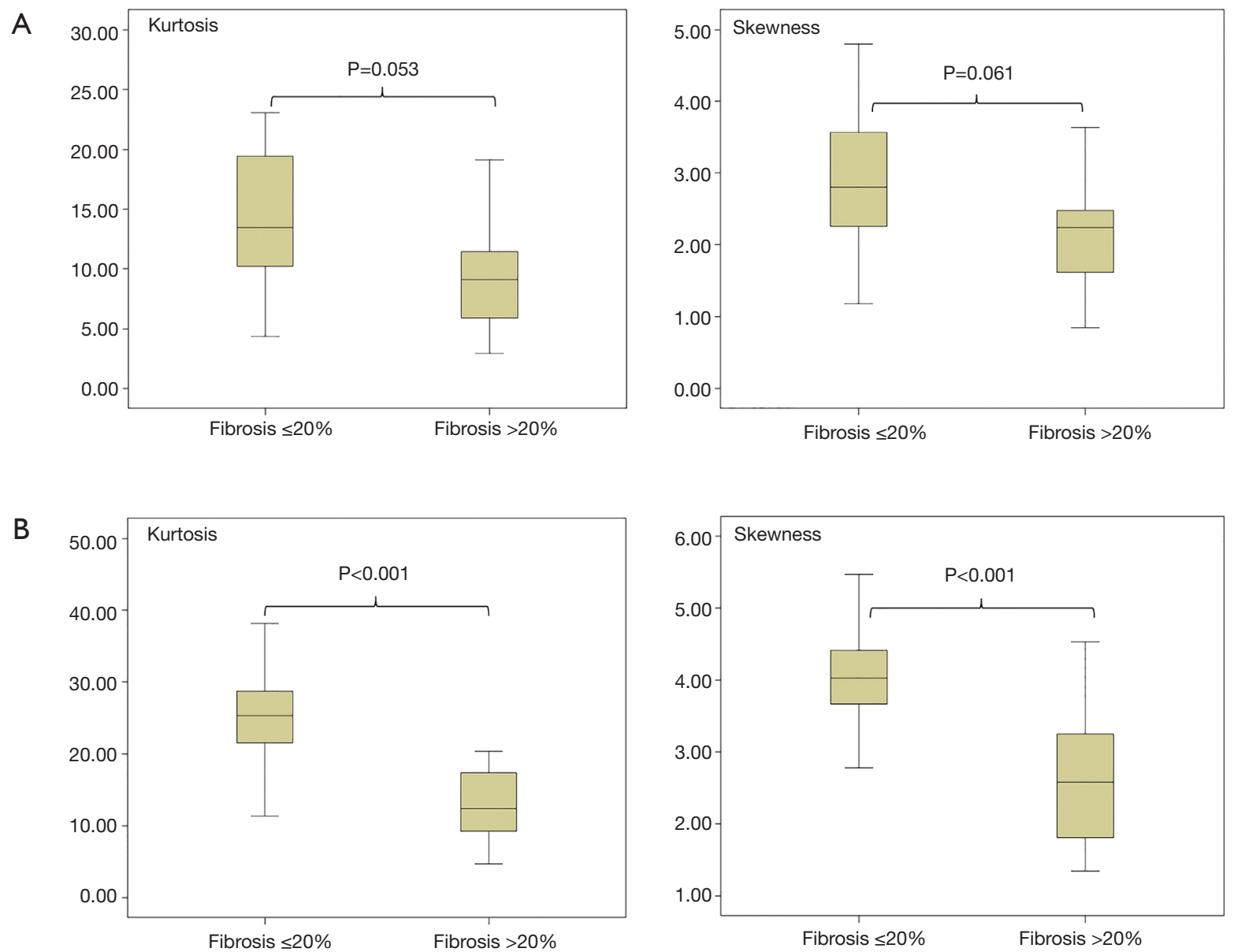

Figure 2 Histogram parameters in correlation with 20\%-cutoff lung fibrosis as a prognostic factor in SSc patients. (A) Kurtosis and skewness of standard HRCT compared to the extend of 20\% fibrosis [group 1: $\leq 20 \%$ fibrosis ( $n=31$ ), group 2: $>20 \%$ fibrosis ( $n=29$ )]; (B) Kurtosis and skewness of reduced HRCT compared to the extent of $20 \%$ fibrosis [group 1: $\leq 20 \%$ fibrosis ( $n=31$ ), group 2: $>20 \%$ fibrosis (n=29)]. HRCT, high-resolution computed tomography.

9.14 versus $12.5 \pm \mathrm{SD} 7.12$, Skewness: mean $3.71 \pm \mathrm{SD} 1.84$ versus $2.58 \pm \mathrm{SD} 0.9 ; \mathrm{P}<0.001$ for both).

\section{Discussion}

This study aimed to assess whether such a dedicated CT as the reduced HRCT with minimal dose can be used for qualitative and quantitative assessment of lung involvement in SSc patients, with special focus on visual assessment and the histogram-based approach in comparison to standard HRCT.

With respect to the detection of parenchymal 
abnormalities, only the detection of intrapulmonary bronchiectasis was significantly lower in the reduced HRCT compared to standard HRCT. This finding may have been caused by the use of the small limited number of thin sliced sequential CT images, as even on standard HRCT the distinguishing of cystic change as honeycombing from bronchiectasis can be difficult (42). Since it is known that traction bronchiectasis is commonly associated with advanced fibrotic changes $(43,44)$ and correlates with the amount of fibrosis (45), it can be assumed, that its detection rate may not influence the overall fibrosis assessment compared to other findings of advanced fibrosis such as honeycombing, coarse reticular pattern, and architectural distortion $(43,44)$.

On the other hand, despite the difference in detection of bronchiectasis no difference in the qualitative and semiquantitative assessment of fibrosis scoring comparing standard and reduced HRCT was seen. Takei et al. could demonstrate in confirmation of known evidence $(11,12)$ that a high fibrosis extension score is associated with an increased risk of death, and further independent from HRCT pattern (13). In this context reduced HRCT might qualify for the visual scoring of lung fibrosis extension as no difference to standard HRCT was found-and could be used as a follow-up examination in monitoring and therapy management of patients with SSc-ILD.

However, the reduced HRCT might miss other lung pathologies, as for example lung cancer. Because the aim of this type of CT protocol is detection and quantification of SSc-ILD and not early lung cancer detection, a wholechest-CT examination at baseline should be recommended to capture incidental intra-parenchymal lesions that might need further follow up. However, in younger patients with expected longer span of life follow up examinations additionally whole-chest CT examinations in bigger time intervals should be considered.

Our results show that quantitative histogram parameters derived from a dedicated reduced HRCT were significantly higher compared to standard HRCT of the whole lung. This finding can be explained by the defined distribution of the slices for the reduced HRCT. By selection of a disproportionally higher number of slices at the lung base, the known less affected parts of the lung for ILD in SSc patients are systematically excluded. This leads to a disproportionate higher evaluation of the affected areas in comparison to the histogram parameters derived from standard HRCT which include also the complete volume of the healthy not affected lung. Especially in early stage SSc patients with only discrete fibrotic intrapulmonary patterns in the lung base such a dedicated reduced HRCT can provide augmented histogram parameters, in which even discreet dynamic changes of fibrotic lung involvement may be represented.

Although histogram parameters derived from both reduced and standard HRCT correlated with the corresponding visual assessment scores for fibrosis severity and extension with no significant difference, only for the histogram parameters from reduced HRCT a discrimination at a threshold of $20 \%$ fibrosis was found with a high sensitivity of $88 \%$ for kurtosis and skewness and a specificity of $81 \%$ for skewness and $86 \%$ for kurtosis resulting in good NPVs of $78-80 \%$ and a moderate PPVs of $62 \%$. Following this approach of a reduced HRCT with focused apico-basal gradient we believe, that in particularly subtle changes for example in ground glass pattern may be earlier quantitatively detected especially in early stage SSc patients during follow up examinations, which even an experienced radiologic eye possibly could miss. Particularly in SSc patients with rapid disease progression one important key parameter is to detect increasing ILD at the earliest time point as possible, and not the general detection of ILD alone, because in this situation immediately progressive treatment initiation is recommended by the experts $(10,46)$. In this context Mendoza et al. even recommend the performance of standard HRCT every 6-8 months for the first 2 years after time point of diagnosis (46), assuming a higher benefit for the patient in case of early detection of disease progression compared to radiation dose protection.

Therefore, the reduced HRCT may represent a powerful tool that not only allows to visualize early ILD in SSc as shown recently $(28,31)$, but may also be a tool to quantify subtle new fibrotic changes in lung parenchyma during follow up, especially with a significantly lower radiation exposure with a $97.4 \%$ reduction of estimated effective radiation dose in our cohort with $0.041 \mathrm{mSv}$ which is in a range of a conventional chest $\mathrm{X}$-ray and can be considered as a screening method for early detection and early evaluation of SSc ILD increase, rather than detection of lung fibrosis alone.

Although FVC correlated significantly with the histogram parameters of standard and reduced HRCT no cut off value for the $20 \%$ lung fibrosis was found. The reason therefore might be differences in population, as most of our cases had early SSc, which are not visible within the limitations of pulmonary function. This concludes that the 
histogram parameters, derived from the reduced HRCT, might be a more robust marker in the assessment of fibrosis in early SSc patients than the clinical parameter FVC, derived from spirometry. The latter has several limitations: it is related to patient's motivation, and has a low sensitivity, and therefore can be normal in case of known minor fibrotic pulmonary changes (17). This was confirmed in a previous study in which high rates of false-negative results in pulmonary function tests in the early detection of ILD screening in SSc patients was observed (7).

Our study has certain limitations:

(I) The major limitation is that we retrospectively reconstructed the reduced HRCT from the standard HRCT and therefore have no technical obstacles, which may occur when performing such a CT protocol prospectively. We tried to simulate the reproducibility of the results derived from the reduced HRCT for both, the visual analysis as well as the quantitative analysis by retrospectively simulating slightly different slices for the reduced HRCT but with the same increment levels to simulate different inspiration depths. Although we could show no significant differences between the retrospectively standardized reconstructed reduced HRCT and the reduced HRCT with slightly different slices in our cohort, our study still faces the limitation of possible breathing artefacts during the examination or overlying of other diseases such as infection or pulmonary congestion. In case of new observed overlying disease, we would recommend comparison of reduced HRCT with recent imaging if available in combination with further clinical examination including additional imaging examination if needed to achieve an appropriate diagnosis for optimal patient treatment. After successful therapy a repetition of reduced HRCT for monitoring of ILD can be considered. An ongoing study will assess the accuracy and robustness of this CT-protocol for follow-up examination of fibrotic changes in the lung in SSc patients.

(II) With the concept of reduced HRCT the detection rate of bronchiectasis was low, which possibly could be explained, that with the use of a small limited number of thin sliced sequential CT images the known difficulties of differentiating bronchiectasis from honeycombing might have been accentuated. Since bronchiectasis is an intraparenchymal pattern associated with advanced fibrotic changes, we believe that our approach of reduced HRCT might rather have its strength in early detection and early recognition of ILD progression. To evaluate these further prospective studies are needed.

(III) Our findings cannot be transferred easily to other diseases with different parenchymal lung involvement. SSc patients develop ILD with frequent NSIP and less often UIP pattern, and in addition have a predominant apico-basal distribution. Furthermore it is known that a small subgroup of patients with NSIP may have a peribronchovascular and upper field distribution of lung fibrosis (47). Additionally the incidence of a small SSc patient number with organizing pneumonia (OP) and combined pulmonary fibrosis and emphysema (CPFE) has been reported (48). As we could not find such intrapulmonary findings in our patient cohort, we were not able to evaluate this aspect. Thus, this technique needs validation in other diseases and further exploration before it can be clinically applied to these conditions.

In conclusion, the reduced HRCT is a robust method to assess lung fibrosis in SSc-ILD with minimal dose. In contrast to standard HRCT histogram parameters derived from the approach of reduced HRCT could discriminate at a threshold of $20 \%$ lung fibrosis with high sensitivity and specificity. Hence it might be used to detect early disease progression of lung fibrosis in context of monitoring and treatment of SSc patients. It therefore has the potential to replace other imaging techniques such as conventional chest $\mathrm{X}$-ray and high dose standard HRCT.

\section{Acknowledgements}

This work was supported by "LUNGE Zürich".

\section{Footnote}

Conflicts of Interest: The authors have no conflicts of interest to declare.

Ethical Statement: Institutional review board approval was obtained (EK \#839) prior to the conduct of this study. All patients signed informed consent.

\section{References}

1. Molberg O, Hoffmann-Vold AM. Interstitial lung disease 
in systemic sclerosis: progress in screening and early diagnosis. Curr Opin Rheumatol 2016;28:613-8.

2. D'Angelo WA, Fries JF, Masi AT, et al. Pathologic observations in systemic sclerosis (scleroderma). A study of fifty-eight autopsy cases and fifty-eight matched controls. Am J Med 1969;46:428-40.

3. Goldin JG, Lynch DA, Strollo DC, et al. Highresolution CT scan findings in patients with symptomatic scleroderma-related interstitial lung disease. Chest 2008;134:358-67.

4. LeRoy EC, Medsger TA Jr. Criteria for the classification of early systemic sclerosis. J Rheumatol 2001;28:1573-6.

5. Lynch DA, Godwin JD, Safrin S, et al. High-resolution computed tomography in idiopathic pulmonary fibrosis: diagnosis and prognosis. Am J Respir Crit Care Med 2005;172:488-93.

6. Remy-Jardin M, Remy J, Wallaert B, et al. Pulmonary involvement in progressive systemic sclerosis: sequential evaluation with CT, pulmonary function tests, and bronchoalveolar lavage. Radiology 1993;188:499-506.

7. Suliman YA, Dobrota R, Huscher D, et al. Brief Report: Pulmonary Function Tests: High Rate of FalseNegative Results in the Early Detection and Screening of Scleroderma-Related Interstitial Lung Disease. Arthritis Rheumatol 2015;67:3256-61.

8. Wells AU, Hansell DM, Corrin B, et al. High resolution computed tomography as a predictor of lung histology in systemic sclerosis. Thorax 1992;47:738-42.

9. Best AC, Meng J, Lynch AM, et al. Idiopathic pulmonary fibrosis: physiologic tests, quantitative CT indexes, and CT visual scores as predictors of mortality. Radiology 2008;246:935-40.

10. Hoffmann-Vold AM, Aalokken TM, Lund MB, et al. Predictive value of serial high-resolution computed tomography analyses and concurrent lung function tests in systemic sclerosis. Arthritis Rheumatol 2015;67:2205-12.

11. Elhai M, Meune C, Boubaya M, et al. Mapping and predicting mortality from systemic sclerosis. Ann Rheum Dis 2017;76:1897-905.

12. Goh NS, Desai SR, Veeraraghavan S, et al. Interstitial lung disease in systemic sclerosis: a simple staging system. Am J Respir Crit Care Med 2008;177:1248-54.

13. Takei R, Arita M, Kumagai S, et al. Radiographic fibrosis score predicts survival in systemic sclerosis-associated interstitial lung disease. Respirology 2018;23:385-91.

14. Camiciottoli G, Orlandi I, Bartolucci M, et al. Lung CT densitometry in systemic sclerosis: correlation with lung function, exercise testing, and quality of life. Chest
2007;131:672-81.

15. Best AC, Lynch AM, Bozic CM, et al. Quantitative CT indexes in idiopathic pulmonary fibrosis: relationship with physiologic impairment. Radiology 2003;228:407-14.

16. Sverzellati N, Zompatori M, De Luca G, et al. Evaluation of quantitative CT indexes in idiopathic interstitial pneumonitis using a low-dose technique. Eur J Radiol 2005;56:370-5.

17. Colombi D, Dinkel J, Weinheimer O, et al. Visual vs Fully Automatic Histogram-Based Assessment of Idiopathic Pulmonary Fibrosis (IPF) Progression Using Sequential Multidetector Computed Tomography (MDCT). PLoS One 2015;10:e0130653.

18. Hoyles RK, Ellis RW, Wellsbury J, et al. A multicenter, prospective, randomized, double-blind, placebo-controlled trial of corticosteroids and intravenous cyclophosphamide followed by oral azathioprine for the treatment of pulmonary fibrosis in scleroderma. Arthritis Rheum 2006;54:3962-70.

19. Tashkin DP, Elashoff R, Clements PJ, et al. Cyclophosphamide versus placebo in scleroderma lung disease. N Engl J Med 2006;354:2655-66.

20. Goldin J, Elashoff R, Kim HJ, et al. Treatment of scleroderma-interstitial lung disease with cyclophosphamide is associated with less progressive fibrosis on serial thoracic high-resolution CT scan than placebo: findings from the scleroderma lung study. Chest 2009;136:1333-40.

21. Kim HJ, Brown MS, Elashoff R, et al. Quantitative texture-based assessment of one-year changes in fibrotic reticular patterns on HRCT in scleroderma lung disease treated with oral cyclophosphamide. Eur Radiol 2011;21:2455-65.

22. Delorme S, Keller-Reichenbecher MA, Zuna I, et al. Usual interstitial pneumonia. Quantitative assessment of highresolution computed tomography findings by computerassisted texture-based image analysis. Invest Radiol 1997;32:566-74.

23. Neroladaki A, Botsikas D, Boudabbous S, et al. Computed tomography of the chest with model-based iterative reconstruction using a radiation exposure similar to chest $\mathrm{X}$-ray examination: preliminary observations. Eur Radiol 2013;23:360-6.

24. Brenner DJ, Hall EJ. Computed tomography--an increasing source of radiation exposure. $N$ Engl J Med 2007;357:2277-84.

25. Brenner DJ. Radiation risks potentially associated with low-dose CT screening of adult smokers for lung cancer. 
Radiology 2004;231:440-5.

26. Radiological protection and safety in medicine. A report of the International Commission on Radiological Protection. Ann ICRP 1996;26:1-47.

27. Pontana F, Billard AS, Duhamel A, et al. Effect of Iterative Reconstruction on the Detection of Systemic Sclerosisrelated Interstitial Lung Disease: Clinical Experience in 55 Patients. Radiology 2016;279:297-305.

28. Winklehner A, Berger N, Maurer B, et al. Screening for interstitial lung disease in systemic sclerosis: the diagnostic accuracy of HRCT image series with high increment and reduced number of slices. Ann Rheum Dis 2012;71:549-52.

29. Bankier AA, Mehrain S, Kienzl D, et al. Regional heterogeneity of air trapping at expiratory thin-section CT of patients with bronchiolitis: potential implications for dose reduction and CT protocol planning. Radiology 2008;247:862-70.

30. de Jong PA, Nakano Y, Lequin MH, et al. Dose reduction for CT in children with cystic fibrosis: is it feasible to reduce the number of images per scan? Pediatr Radiol 2006;36:50-3.

31. Frauenfelder T, Winklehner A, Nguyen TD, et al. Screening for interstitial lung disease in systemic sclerosis: performance of high-resolution CT with limited number of slices: a prospective study. Ann Rheum Dis 2014;73:2069-73.

32. Meier FM, Frommer KW, Dinser R, et al. Update on the profile of the EUSTAR cohort: an analysis of the EULAR Scleroderma Trials and Research group database. Ann Rheum Dis 2012;71:1355-60.

33. Minier T, Guiducci S, Bellando-Randone S, et al. Preliminary analysis of the very early diagnosis of systemic sclerosis (VEDOSS) EUSTAR multicentre study: evidence for puffy fingers as a pivotal sign for suspicion of systemic sclerosis. Ann Rheum Dis 2014;73:2087-93.

34. Preliminary criteria for the classification of systemic sclerosis (scleroderma). Subcommittee for scleroderma criteria of the American Rheumatism Association Diagnostic and Therapeutic Criteria Committee. Arthritis Rheum 1980;23:581-90.

35. AAoPi M. The Measurement, Reporting, and Management of Radiation Dose in CT: Report of AAPM Task Group 23 of the Diagnostic Imaging Council CT Committee. American Association of Physicists in Medicine 2008.

36. Moore OA, Goh N, Corte T, et al. Extent of disease on high-resolution computed tomography lung is a predictor of decline and mortality in systemic sclerosis- related interstitial lung disease. Rheumatology (Oxford) 2013;52:155-60.

37. Hayes AF. Answering the call for a standard reliability measure for coding data. Commun Methods Meas 2007;1:77-89.

38. Krippendorff K. Content Analysis: An Introduction to Its Methodology. 2004;2nd ed.

39. Krippendorff K. Reliability in Content Analysis: Some Common Misconceptions and Recommendations. Human Communication Research 2004;30:411-33.

40. Walker UA, Tyndall A, Czirjak L, et al. Clinical risk assessment of organ manifestations in systemic sclerosis: a report from the EULAR Scleroderma Trials And Research group database. Ann Rheum Dis 2007;66:754-63.

41. Badesch DB, Champion HC, Sanchez MA, et al. Diagnosis and assessment of pulmonary arterial hypertension. J Am Coll Cardiol 2009;54:S55-66.

42. Devaraj A. Imaging: how to recognise idiopathic pulmonary fibrosis. Eur Respir Rev 2014;23:215-9.

43. Jawad H, Chung JH, Lynch DA, et al. Radiological approach to interstitial lung disease: a guide for the nonradiologist. Clin Chest Med 2012;33:11-26.

44. Nishimura K, Kitaichi M, Izumi T, et al. Usual interstitial pneumonia: histologic correlation with high-resolution CT. Radiology 1992;182:337-42.

45. Johkoh T, Muller NL, Colby TV, et al. Nonspecific interstitial pneumonia: correlation between thin-section CT findings and pathologic subgroups in 55 patients. Radiology 2002;225:199-204.

46. Mendoza FA, Mansoor M, Jimenez SA. Treatment of Rapidly Progressive Systemic Sclerosis: Current and Futures Perspectives. Expert Opin Orphan Drugs 2016;4:31-47.

47. Hartman TE, Swensen SJ, Hansell DM, et al. Nonspecific interstitial pneumonia: variable appearance at highresolution chest CT. Radiology 2000;217:701-5.

48. Morales-Cardenas A, Perez-Madrid C, Arias L, et al. Pulmonary involvement in systemic sclerosis. Autoimmun Rev 2016;15:1094-108.

Cite this article as: Nguyen-Kim TD, Maurer B, Suliman YA, Morsbach F, Distler O, Frauenfelder T. The impact of slice-reduced computed tomography on histogram-based densitometry assessment of lung fibrosis in patients with systemic sclerosis. J Thorac Dis 2018;10(4):2142-2152. doi: 10.21037/jtd.2018.04.39 


\section{Supplementary}

Table S1 Fibrosis severity score (range, 0 to $15^{*}$ ) adapted from Camiciottoli et al. (14)

\begin{tabular}{lc}
\hline Parenchymal abnormality & Score \\
\hline Ground-glass appearance & 1 \\
Irregular pleural margins & 2 \\
Septal and subpleural lines & 3 \\
Honeycombing & 4 \\
Subpleural cysts & 5 \\
\hline
\end{tabular}

*, example: in case of presence of all five lung abnormalities all scores are summed to an overall score of 15 (score $1+$ score $2+$ score $3+$ score $4+$ score 5 = overall fibrosis severity score 15 ).
Table S2 Fibrosis extension score (range, 0 to $24^{*}$ ) adapted from Goldin et al. (3)

Upper zone: from apex to upper level of carina

Middle zone: from upper part of carina to division of main lower lobe bronchus into segmental bronchi

Lower zone: from the division of main lower lobe bronchus into segmental bronchi to the basis of the lung

Scale from $0-4$ : 0 , no fibrosis; $1,1 \%$ to $25 \%$; $2,26 \%$ to $50 \%$; $3,51 \%$ to $75 \% ; 4,76 \%$ to $100 \%$. ${ }^{*}$, example: in case of a maximum fibrosis score 4 (76\% to $100 \%)$ in each of the three zones of one lung side the scores are summed to an overall fibrosis extension score 12, if both lungs would be affect symmetrically, the sum of the overall score would be 24 . 